\section{Computational molecular modelling as a platform for a deeper understanding of protein dynamics and rational drug design}

\author{
Gianvito Grasso, ${ }^{1}$ Lorenzo Pallante, ${ }^{2}$ \\ Jack A. Tuszynski, 2
}

Umberto Morbiducci, 2 Marco A. Deriu ${ }^{2}$

1 Istituto Dalle Molle di Studi sull'Intelligenza Artificiale (IDSIA), Scuola Universitaria Professionale della Svizzera Italiana (SUPSI), Università della Svizzera Italiana (USI), Centro Galleria 2, Manno, Switzerland; 2Department of Mechanical and Aerospace Engineering, Politecnico di Torino, Turin, Italy

\begin{abstract}
Elucidating structural features of protein aggregation at molecular level may provide novel opportunities for overarching therapeutic approaches such as blocking common aggregation-induced cellular toxicity pathways. In this context molecular modelling stimulates further research on amyloid aggregation modulators and modelling platforms can be used to test the efficiency of potential aggregation inhibitors aimed at destabilizing/reducing the stability of the amyloidogenic proteins.
\end{abstract}

\section{Introduction}

Several possible alternatives to the use of animal models have been suggested: in vitro models, cell cultures, engineered tissues, and computer models. In this context the use of sophisticated molecular and multiscale computational models may help to understand mechanisms driving physiological and pathological behavior of complex biological systems. Computational molecular modelling represents a promising prospect for advances in a number of research fields. For example, in neurodegenerative diseases, understanding molecular mechanisms driving protein function/misfunction due to misfolding may lead to rational drug design strategies based on a deeper knowledge of protein structurefunction relationships. Whereas a complete replacement of animals by computer models in the field of drug design is today only a dream, the use of molecular modelling techniques have already allowed to signifi- cantly reduce the experimental efforts in terms of resources and costs of experimentation.

\section{Materials and Methods}

Molecular modelling consists of theoretical methods and computational techniques able to describe the behaviour of complex biochemical systems (e.g., membranes, proteins, small molecules) and their interactions by quantum and classical physics. Whereas approaches considering the quantum level ( $a b$ initio approaches) provide the most detailed analysis of molecular systems, they are limited by system size and complexity and cannot yet provide enough information to investigate dynamics of proteins or protein assemblies. Molecular Mechanics and Molecular Dynamics (MD) ignore the electronic degrees of freedom and focus the modelling on nuclear positions and a classical description of atomic interactions. MD produces a molecular trajectory with atomic resolution by the iterative integration in time of Newton's equations of motion for all atomic degrees of freedom. While exploring the time evolution of the molecular system, MD is able to capture thermodynamic properties (directly comparable with experimental data) by sampling all accessible conformational microstates in a defined statistical ensemble. Due to above mentioned peculiar features, MD is becoming a relevant player in the neurodegenerative research field, being able to describe protein tendencies towards specific conformational rearrangements and protein-protein organization. Approaching this problem from an energetic point of view is of great importance especially in case of amyloidogenic proteins, given the intimate interconnection between the functional energy landscape and aggregation risk. A deeper knowledge on the abovementioned interconnection may significantly support the design of effective pharmacological and non-pharmacological strategies capable of driving misfolded or mis-aggregated proteins toward physiological conditions and away from pathological states.

\section{Results}

Approaches such as classical and enhanced MD have already been employed to provide a quantitative estimation of the free energy landscape characterizing the early stages of protein aggregation responsible for the onset of different diseases, such as Spinocerebellar Ataxia Type-1 (SCA1) ${ }^{1,2}$
Correspondence: Marco A. Deriu, Department of Mechanical and Aerospace Engineering, Politecnico di Torino, Turin, Italy.

E-mail: marco.deriu@polito.it

Key words: Molecular modelling; drug design neurodegenerative; protein folding.

Conference presentation: this paper was presented at the Second Centro 3R Annual Meeting - 3Rs in Italian Universities, 2019, June 20-21, University of Genoa, Italy.

Received for publication: 28 October 2019. Accepted for publication: 6 November 2019

This work is licensed under a Creative Commons Attribution NonCommercial 4.0 License (CC BY-NC 4.0).

CCopyright: the Author(s), 2019

Licensee PAGEPress, Italy

Biomedical Science and Engineering 2019; 3(s2):87 doi:10.4081/bse.2019.87

and Alzheimer's Disease (AD). ${ }^{3-5}$ More in detail, classical and enhanced sampling techniques have recently fully characterized the AXH aggregation pathway from monomer to tetramer, highlighting several protein mutations responsible for the destabilization of the monomer/dimer/tetramer equilibrium states. Residues identified as mainly responsible for dimer-dimer interactions may be considered as an important target space for pharmacological intervention. Computational modelling has also provided fruitful insight into the conformational dynamics and stability characterizing $\mathrm{A} \beta$ fibrils, represented by a polymorphic structure and responsible for the onset of Alzheimer's Disease. For example, evidence about the stability of the recently proposed S-shaped A $\beta$ fibril model, has been provided by computational simulations. ${ }^{3,5}$ The S-shaped architecture shows superior mechanical performance compared to the U-shaped motif due to a better distribution of the mechanical stress, indicating the importance of molecular architecture in modulating the conformational dynamics and stability of the protein assembly. This aspect must be taken into account when designing inhibitors, which should hit all achievable pathologic forms of $A \beta$ fibrils (Figure 1).

\section{Conclusions}

Elucidating the common structural features of protein aggregation diseases at molecular level may provide novel opportu- 
nities for overarching therapeutic approaches such as inhibiting aggregation or blocking common aggregation-induced cellular toxicity pathways. To address this issue, it is extremely important to shed light on the molecular interactions responsible for protein aggregation. Information obtained from molecular modelling stimulates further research on amyloid aggregation modulators and modelling platforms can be used to test the efficiency of potential aggregation inhibitors aimed at destabilizing/reducing the stability of the amyloidogenic proteins. A tight feedback loop between molecular modeling predictions and experimental validation will become the future backbone of successful drug discovery strategies.

\section{References}

1. Grasso G, Morbiducci U, Massai D, et al. Destabilizing the AXH Tetramer by Mutations: Mechanisms and Potential Antiaggregation Strategies. Biophys J 2018;114:323-30.

2. Deriu MA, Grasso G, Tuszynski JA, et al. Characterization of the AXH domain of Ataxin-1 using enhanced sampling and functional mode analysis. Proteins 2016;84:666-73.

3. Grasso G, Rebella M, Muscat S, et al.

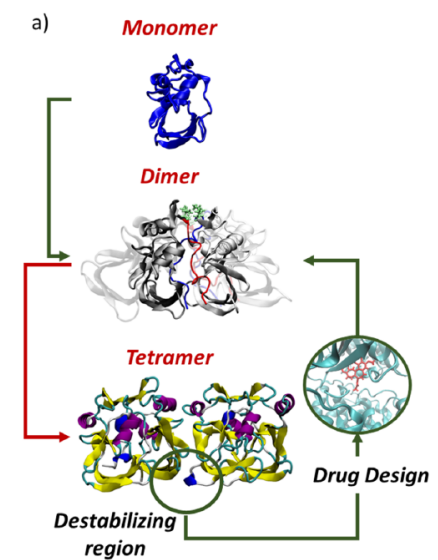

b)

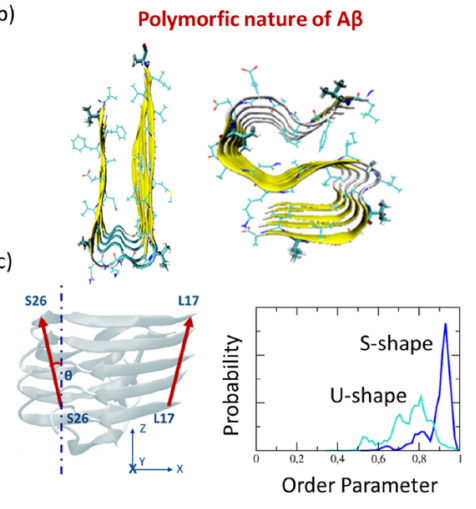

Figure 1. a) AXH monomer/dimer/tetramer. A better understanding of the protein aggregation dynamics drives the drug design; b) Polymorphic nature of $A \beta$ amyloids; c) metrics to investigate their conformational stability. Effective compounds should be designed to destabilize all possible fibril arrangements.

Conformational Dynamics and Stability of U-Shaped and S-Shaped Amyloid $\beta$ Assemblies. Int J Mol Sci 2018; 19:E571.

4. Dal Magro R, Simonelli S, Cox A, et al. The Extent of Human Apolipoprotein A-I Lipidation Strongly Affects the $\beta$ Amyloid Efflux Across the Blood-Brain
Barrier in vitro. Front Neurosci 2019;13:419.

5. Grasso G, Rebella M, Morbiducci U, et al. The Role of Structural Polymorphism in Driving the Mechanical Performance of the Alzheimer's Beta Amyloid Fibrils. Front Bioeng Biotechnol 2019;7:83. 